\title{
Associations between $A P O E$ and low-density lipoprotein cholesterol genotypes and cognitive and physical capability: the HALCyon programme
}

\author{
Tamuno Alfred • Yoav Ben-Shlomo • Rachel Cooper • Rebecca Hardy • \\ Cyrus Cooper • Ian J. Deary • Jane Elliott • David Gunnell • \\ Sarah E. Harris • Mika Kivimaki • Meena Kumari • Richard M Martin • \\ Chris Power • Avan Aihie Sayer • John M. Starr • Diana Kuh • \\ Ian NM Day • the HALCyon Study Team
}

Received: 2 September 2013 / Accepted: 18 June 2014 / Published online: 30 July 2014

(C) The Author(s) 2014. This article is published with open access at Springerlink.com

\begin{abstract}
The $A P O E$ \&2/3/4 genotype has been associated with low-density lipoprotein cholesterol (LDL-C) and Alzheimer disease. However, evidence for associations with measures of cognitive performance in adults without dementia has been mixed, as it is for physical performance. Associations may also be evident in other genotypes implicated in LDL-C levels. As part of the Healthy Ageing across the Life Course (HALCyon) collaborative research programme, genotypic information was obtained for $A P O E \varepsilon 2 / 3 / 4$, rs515135 (APOB), rs2228671 (LDLR) and rs629301 (SORT1) from eight cohorts of adults aged between 44 and $90+$ years. We
\end{abstract}

Electronic supplementary material The online version of this article (doi:10.1007/s11357-014-9673-9) contains supplementary material, which is available to authorized users.

T. Alfred $(\bowtie) \cdot$ Y. Ben-Shlomo • D. Gunnell • R. M. Martin • I. N. Day

School of Social and Community Medicine, University of Bristol, Canynge Hall, 39 Whatley Road, Bristol BS8 2PS, UK

e-mail: tamuno.alfred@bristol.ac.uk

R. Cooper $\cdot$ R. Hardy $\cdot$ D. Kuh

MRC Unit for Lifelong Health and Ageing, University

College London, London WC1B 5JU, UK

C. Cooper $\cdot$ A. A. Sayer

MRC Lifecourse Epidemiology Unit, University of

Southampton, Southampton SO16 6YD, UK

C. Cooper

Institute of Musculoskeletal Sciences, University of Oxford,

Oxford OX3 7LD, UK investigated associations with four measures of cognitive (word recall, phonemic fluency, semantic fluency and search speed) and physical capability (grip strength, get up and go/walk speed, timed chair rises and ability to balance) using meta-analyses. Overall, little evidence for associations between any of the genotypes and measures of cognitive capability was observed (e.g. pooled beta for $A P O E \& 4$ effect on semantic fluency $z$ score $=$ $-0.02 ; 95 \% \mathrm{CI}=-0.05$ to $0.02 ; p$ value $=0.3 ; n=$ 18,796). However, there was borderline evidence within studies that negative effects of $A P O E \& 4$ on nonverbal ability measures become more apparent with age. Few

\author{
I. J. Deary $\cdot$ S. E. Harris $\cdot$ J. M. Starr \\ Centre for Cognitive Ageing and Cognitive Epidemiology, \\ University of Edinburgh, Edinburgh EH8 9JZ, UK \\ I. J. Deary \\ Department of Psychology, University of Edinburgh, \\ Edinburgh EH8 9JZ, UK \\ J. Elliott \\ Centre for Longitudinal Studies, Department of Quantitative \\ Social Sciences, Institute of Education, 20 Bedford Way, \\ London WC1H 0AL, UK \\ S. E. Harris \\ Medical Genetics Section, Molecular Medicine Centre, \\ Institute of Genetics and Molecular Medicine, Western \\ General Hospital, Crew Road, Edinburgh EH4 2XU, UK
}


genotypic associations were observed with physical capability measures. The findings from our large investigation of middle-aged to older adults in the general population suggest that effects of APOE on cognitive capability are at most modest and are domain- and agespecific, while $A P O E$ has little influence on physical capability. In addition, other LDL-C-related genotypes have little impact on these traits.

Keywords Ageing · Apolipoprotein E - Cognition · Single nucleotide polymorphism

\section{Introduction}

Higher levels of low-density lipoprotein cholesterol (LDL-C) have been associated with a number of agerelated conditions, including increased risk of coronary heart disease (Wilson et al. 1998). Although modification by diet (Kelley et al. 2012) and pharmacological interventions (Law et al. 2003) is possible, LDL-C is partly heritable (Matteini et al. 2010), with several identified genetic variants contributing to its interindividual variability. Among these, the $\varepsilon 2, \varepsilon 3$ and $\varepsilon 4$ alleles of the $A P O E$ gene are probably the most widely studied. Encoding apolipoprotein E and playing a key role in lipid metabolism (Mahley and Rall 2000), several studies have observed that carriers of the $A P O E \varepsilon 2$ and $\varepsilon 4$ alleles display lower and higher, respectively, levels of LDL-C compared with the most common $\varepsilon 3 / \varepsilon 3$ genotype (Bennet et al. 2007). In addition, it has been long

M. Kivimaki $\cdot$ M. Kumari

Department of Epidemiology and Public Health, University College London, London WC1E 6BT, UK

R. M. Martin • I. N. Day

MRC Centre for Causal Analyses in Translational

Epidemiology, University of Bristol, Bristol BS8 2BN, UK

C. Power

Centre for Paediatric Epidemiology and Biostatistics, UCL Institute of Child Health, 30 Guilford Street, London WC1N $1 \mathrm{EH}, \mathrm{UK}$

A. A. Sayer

Academic Geriatric Medicine, Southampton General Hospital, University of Southampton, Southampton SO16 6YD, UK

J. M. Starr

Alzheimer Scotland Dementia Research Centre, University of Edinburgh, 7 George Square, Edinburgh EH8 9JZ, UK established that carriers of the $\varepsilon 2$ alleles are at lower risk, and those with the $\varepsilon 4$ alleles are at higher risk of Alzheimer disease (Bertram et al. 2007). Among cognitively healthy individuals, there is also evidence from many, though not all studies, that carriers of the $\varepsilon 4$ allele perform worse in some cognitive tests and that these effects may be more pronounced in older populations (Caselli et al. 2009; Wisdom et al. 2011; Davies et al. 2014). For instance, there is little evidence to support the influence of the $A P O E$ genotype on cognitive measures in children and young people (Taylor et al. 2011; Ihle et al. 2012), though there is some mixed evidence for its influence in middle-aged (Zhao et al. 2005; Bunce et al. 2011) and older (Deary et al. 2004a) adults. In addition, there is some evidence from longitudinal studies that $\varepsilon 4$ carriers experience greater cognitive decline in mid(Blair et al. 2005) and later (Schiepers et al. 2012) life. $A P O E$ may also have a role in ageing phenotypes more generally, with other associations including triglycerides, apolipoproteins, C-reactive protein (Khan et al. 2013), type 2 diabetes (Anthopoulos et al. 2010), longevity (McKay et al. 2011; Nebel et al. 2011) and Parkinson disease (Lill and Roehr).

Given that the observed associations between cognitive and physical performance measures (Deary et al. 2006; Clouston et al. 2013) may be a result of genetic influences on shared age-related pathways simultaneously leading to the impairment of both (Johnson et al. 2009), it has been hypothesised that the pleiotropic $A P O E$ genotype may also affect physical performance. However, to date, investigations into associations between $A P O E$ and physical performance have also been inconsistent, with the $\varepsilon 4$ allele reported to be associated with slower gait speeds (Melzer et al. 2005), but not with fitness (Deary et al. 2006), frailty (Rockwood et al. 2008), or grip strength (Vasunilashorn et al. 2013) and mixed findings for disability (Hyman et al. 1996; Kulminski et al. 2008) and chair stands speed (Melzer et al. 2005; Vasunilashorn et al. 2013) in middle-aged and older adults.

Therefore, further investigations into associations between $A P O E$ and cognitive and physical performance measures are required. It is also of interest to examine whether any such effects are related to an effect on LDLC. Given the conflicting evidence for associations between levels of LDL-C and cognitive (Yaffe et al. 2002; Packard et al. 2007) and physical performance measures (Walston et al. 2002; Blain et al. 2012), investigations of other LDL-C-related genotypes may help to elucidate 
whether or not any effects are unique to $A P O E$. Therefore, we analysed data from 23,916 participants aged between 44 and 90+ from eight UK cohorts as part of the HALCyon research programme (Healthy Ageing across the Life Course; www.halcyon.ac.uk) to investigate associations between the $A P O E$ genotype and objective measures of physical and cognitive capability, the capacity to undertake the physical and mental tasks of daily living. We also explored whether any effects are also demonstrated for other genotypes robustly associated with LDL-C (rs515135 (APOB), rs2228671 (LDLR) and rs629301 (SORT1)) in genome-wide association studies (Aulchenko et al. 2009; Teslovich et al. 2010) (GWAS).

\section{Methods}

Study populations

Participants from eight UK cohorts of the HALCyon programme were included in this report. The National Child Development Study (NCDS) has followed up all individuals born in England, Scotland and Wales during 1 week in March 1958. In 2002-2004, a Biomedical Survey was conducted during home visits by a research nurse. Following informed consent, DNA was extracted from 8,017 participants aged 44-45 years; the sample with immortalised cell line culture $(n=7,526)$ is used here. In 2008-2009, an eighth sweep was carried out during which cognitive performance tests were conducted (Brown and Dodgeon 2010). Further details of the study are available elsewhere (Power and Elliott 2006).

The Medical Research Council National Survey of Health and Development (NSHD) comprises participants sampled from all births in a week in March 1946 and followed up since. In 1999, at age 53 years, men and women were visited by a research nurse and DNA was extracted from 2,756 members of the cohort. Details of the data collected and the several phases of the study are available on the cohort's website (www.nshd.mrc.ac.uk) and elsewhere (Wadsworth et al. 2006).

The Whitehall II study targeted all civil servants aged between 35 and 55 years working in London in 19851988. In 2002-2004 (phase 7), the genetics study was established and DNA was extracted from 6,156 participants. Details of the study design and data collected have been described elsewhere (Marmot and Brunner 2005).

The Caerphilly Prospective Study (CaPS) recruited 2,512 men aged between 45 and 59 years in 1979-1983 from the town of Caerphilly, South Wales and its surrounding villages. Blood samples were collected at baseline and at each of the four follow-ups (phase II, 1984-1988; phase III, 19891993; phase IV, 1993-1997 and phase V, 20022004). Further details are available on the cohort's website (http://www.bris.ac.uk/social-communitymedicine/projects/caerphilly).

The English Longitudinal Study of Ageing (ELSA) comprises men and women aged 50 years and over who originally participated in the Health Survey for England in 1998, 1999 or 2001. Fieldwork began in 2002-2003 (phase I) with two yearly follow-ups, and in 2004-2005 (phase II), blood samples were provided by 6,231 participants. Details of the cohort have been published elsewhere (Marmot et al. 2003).

The Hertfordshire Cohort Study (HCS) consists of 2,997 participants born 1931-1939 and registered with a general practitioner in East, North or West Hertfordshire who attended a clinic in 19942004 (phase I). A second assessment took place in 2004-2005 for participants in East Hertfordshire (phase II). Further details of the study design, data collected and summaries of participant characteristics have been published (Syddall et al. 2005) and are available on its website (www.mrc.soton.ac.uk/ herts).

The Boyd Orr cohort is a historical cohort study based on children surveyed in 1937-1939 in English and Scottish districts. Participants were followed up for vital status via the NHS Medical Information Research Service (MIRS) since 1948, with questionnaire administration to survivors in 1997-1998 (phase II) and a research clinic visit in 2002-2003 (phase III), during which DNA was extracted from 728 adults, of which 397 of 405 who attended specific measurement research clinics had at least one physical capability measure and one relevant genotype called for this analysis. Details of the study design and the data collected have been described on its website (http://www.bris.ac.uk/social-communitymedicine/projects/boyd-orr) and elsewhere (Martin et al. 2005). 
The Lothian Birth Cohort 1921 (LBC1921) participants were all born in 1921 and most completed a cognitive ability assessment at age 11 years. In 1999-2001 (wave I), at approximately 79 years old, 550 participants living in and around Edinburgh, underwent a series of cognitive and physical tests. In 2003-2005 (wave II), 321 returned at 83 years old. Details of the recruitment into the study are available on its website (www. lothianbirthcohort.ed.ac.uk) and have been published previously (Deary et al. 2004b; Deary et al. 2012).

Genotyping and quality control

Single nucleotide polymorphisms (SNPs) rs7412 and rs429358 form the APOE genotype ( $\varepsilon 2 / \varepsilon 2, \varepsilon 2 / \varepsilon 3, \varepsilon 2 /$ $\varepsilon 4, \varepsilon 3 / \varepsilon 3, \varepsilon 3 / \varepsilon 4$ and $\varepsilon 4 / \varepsilon 4 ; 19 q 13.32)$ and genotyping was carried out by KBioscience (www. lgcgenomics.com) in Boyd Orr, CaPS, HCS, NCDS and NSHD. In ELSA, two TaqMan assays (rs429358 and rs7412, Assay-On-Demand, Applied Biosystems, Geneservice Ltd, Cambridge, UK) were used and run on a 7900HT analyzer (Applied Biosystems), and genotypes indicated by the Sequence Detection Software version 2.0 (Applied Biosystems). Details have been described previously in LBC1921 (Schiepers et al. 2012) and Whitehall II (Sabia et al. 2010). Genotyping for other SNPs (rs515135 (APOB, 2p24.1), rs2228671 (LDLR, 19p13.2) and rs629301 (SORT1, 1p13.3)) in ELSA, HCS, CaPS and Boyd Orr were carried out by KBioscience, as well as for rs2228671 in NSHD. In LBC1921, information came from the Illumina Human 610-Quadv1 Chip (using proxies rs541041 for rs515135 and rs646776 for rs629301) (Houlihan et al. 2010). SNPs in NCDS came from two sources-Illumina HumanHap $550 \mathrm{k}$ v3 and Illumina 1.2 $\mathrm{M}$ chips (using rs562338 and rs541041 as proxies for rs515135 and rs646776 as a proxy for rs629301) (Wellcome Trust Case Control Consortium 2007). Genotype information for these three SNPs in Whitehall II as well as rs515135 and rs2228671 in NSHD came from the Illumina Metabochip (www.illumina.com). Genotypic data quality was reviewed by assessing departure from the Hardy-Weinberg equilibrium (HWE), clustering quality (using KBioscience software SNPviewer on their data) and call rates.
Phenotypes

\section{Cognitive capability}

A number of cognitive performance tests in the different studies were used to assess cognitive capability. Different assessments of verbal memory (Bopp and Verhaeghen 2005) were conducted: in ELSA and NCDS, a list of 10 common words were used, with participants asked to recall the list immediately and again after a delay of around $5 \mathrm{~min}$; the mean score was used in the analysis; in NSHD, 15 words were used over three trials; in Whitehall II, 20 words were used; responses in NSHD and Whitehall II were given in writing. In Whitehall II, participants recalled in writing in $1 \mathrm{~min}$ as many words as possible beginning with ' $\mathrm{S}$ ' to assess phonemic fluency (Borkowski et al. 1967), while in LBC1921 three letters 'C', ' $F$ ' and 'L' were used with responses given orally. Participants were asked to recall as many animals as possible within $1 \mathrm{~min}$ to measure semantic fluency (Borkowski et al. 1967); responses were given orally in ELSA, NCDS, CaPS and NSHD, and in writing in Whitehall II. To assess search speed (Richards et al. 1999), 1-min letter searches among grids of letters were used, 600 letters in NSHD and 780 in ELSA and NCDS.

\section{Physical capability}

Grip strength was measured in NSHD, ELSA, HCS and LBC1921 using electronic or hydraulic dynamometers, with the best measure used in the analysis where more than one trial was conducted. Standing balance tests were conducted in the studies, with participants' eyes open: flamingo (up to a maximum time of $30 \mathrm{~s}$ ) (Sport 1993), in NSHD, HCS, Boyd Orr and CaPS, and sideby-side, semi-tandem and full tandem (Stevens et al. 2008) in ELSA. Ability to balance was defined in this analysis as the ability to complete $30 \mathrm{~s}$ of the flamingo or $5 \mathrm{~s}$ of the tandem test. The timed get up and go test (Podsiadlo and Richardson 1991) was carried out in HCS, Boyd Orr and CaPS and required participants to get up from a chair, walk $3 \mathrm{~m}$ at a normal pace, turn, walk back, turn and sit down. Timed walks over $2.44 \mathrm{~m}$ $(8 \mathrm{ft})$ at a normal pace and $6 \mathrm{~m}$ at a fast pace were carried out in ELSA and LBC1921, respectively. Speeds were calculated for timed walks and get up and go, with the fastest speeds used in the analysis where more than one trial was conducted. Timed chair rises (Csuka and 
McCarty 1985) involved asking participants to rise from a chair and sit back down 5 times in ELSA and HCS and 10 times in NSHD; the reciprocal of time taken in seconds $\times 100$ (Kuh et al. 2005) was used in the analysis. Further details of these measurements in these cohorts are presented elsewhere (Cooper et al. 2011).

\section{Statistical methods}

Where information on ethnicity was collected, nonEuropean participants were excluded from analyses in order to avoid confounding from population stratification (Cordell and Clayton 2005). Within studies, linear regression analyses were conducted on the continuous traits and logistic regression analyses were conducted on the ability to balance, adjusting for age, except in the age homogeneous cohorts of NCDS, NSHD and LBC1921 as well as sex in all cohorts except CaPS. APOE genotype was analysed here in two ways: first, $\varepsilon 2+(\varepsilon 2 / \varepsilon 2$ or $\varepsilon 2 / \varepsilon 3)$ and $\varepsilon 4+(\varepsilon 3 / \varepsilon 4$ or $\varepsilon 4 / \varepsilon 4)$ were compared with $\varepsilon 3 /$ $\varepsilon 3$; second, $\varepsilon 4+(\varepsilon 3 / \varepsilon 4$ and $\varepsilon 4 / \varepsilon 4)$ was compared with non $\varepsilon 4(\varepsilon 2 / \varepsilon 2, \varepsilon 2 / \varepsilon 3$ and $\varepsilon 3 / \varepsilon 3)$. Due to the differences observed on LDL-C for carriers of the $\varepsilon 2$ and $\varepsilon 4$ alleles (Bennet et al. 2007), the $\varepsilon 2 / \varepsilon 4$ genotype was excluded from the $A P O E$ analysis. Additive models were used for SNPs rs515135 (APOB), rs2228671 (LDLR) and rs629301 (SORT1), with genotypes coded as 0, 1 and 2 for the number of LDL-raising alleles. Likelihood ratio tests were used to compare the fit of the additive models compared with the full genotype model. Allelic scores were derived from counts of the number of $A P O E$ $\varepsilon 4$ or LDL-C-raising alleles. For continuous traits, the normality of the standardised residuals was inspected with distributional diagnostic plots. For the harmonisation of continuous traits that were used to obtain pooled estimates of the genotypic effects, $z$ score units were calculated in each study by subtracting the study mean and dividing by its standard deviation. The overall mean for $z$ scores is 0 and standard deviation 1 . Two-step (Riley et al. 2010) meta-analyses using a random-effects model were performed to obtain pooled genotypic effects. The $I^{2}$ measure was used to quantify heterogeneity (Higgins et al. 2003). Analyses of the interaction between $\varepsilon 4$ carrier status and age within studies were also conducted. Where outcomes were collected at more than one phase, within-study analyses were performed on the annual change in scores using differences between the most recent and earliest measure available. Finally, the calculation of $z$ scores, for the continuous traits, and the main analyses were repeated in men and women separately. Reporting of the analyses met the appropriate items of recommended checklists (Stroup et al. 2000; Little et al. 2009). A two-tailed significance level of $p<0.05$ was used as evidence of statistical significance. Statistical analysis was performed in Stata 12.1 (StataCorp LP). Quanto (Gauderman and Morrison 2006) was used for power calculations.

\section{Results}

Cohort summaries and genotyping quality

A total of 23,916 adults aged between 44 and 90+years had at least one relevant genotype and phenotype available (Table 1). Allelic frequencies were similar across the studies. Of the 23,061 participants with available APOE data, the genotypic frequencies were as follows: $\varepsilon 2 / \varepsilon 2-0.6 \%, \varepsilon 2 / \varepsilon 3-12.3 \%, \varepsilon 2 / \varepsilon 4-2.6 \%, \varepsilon 3 / \varepsilon 3-$ $58.5 \%, \varepsilon 3 / \varepsilon 4-23.6 \%$ and $\varepsilon 4 / \varepsilon 4-2.4 \%$. The HWE condition was met in all studies for all polymorphisms ( $p$ values $>0.1$ ), except for rs515135 $(A P O B)$ and rs2228671 $(L D L R)$ in NSHD and rs515135 (APOB) in ELSA ( $p$ values $=0.04)$. Summaries of measures of $\operatorname{cog}$ nitive and physical capability are presented in Table S1.

Associations between $A P O E$ genotypes and cognitive capability

Figures S1-S4 present the associations between the cognitive capability measures and $\varepsilon 2+$ and $\varepsilon 4+$, adjusting for age and sex, using $\varepsilon 3 / \varepsilon 3$ as the reference group in the cross-sectional analyses. There was no evidence for associations in the pooled analyses. Within studies, there was evidence for negative effects of $\varepsilon 2+$ and $\varepsilon 4+$ in NSHD and ELSA, respectively, on word recall (Figure S1), and of $\varepsilon 2+$ in ELSA on semantic fluency (Figure S3). Table 2 shows that there was no evidence for associations between $A P O E$ and the cognitive capability measures when comparing carriers of the $\varepsilon 4$ allele with noncarriers. Table S2 presents the findings of the interactions between the $\varepsilon 4$ allele and age within the studies. In ELSA, there was some evidence that a negative effect of $\varepsilon 4$ on word recall increases with age $(p$ value $=0.04)$ and borderline evidence for this on search speed ( $p$ value $=0.08$ ). There was some evidence that the effects of $\varepsilon 4$ allele on word recall differs between men and women ( $p$ value for 
Table 1 Summary of sex, age and LDL-related genotypes by cohort

\begin{tabular}{|c|c|c|c|c|c|c|c|c|c|}
\hline & Cohort & & & & & & & & \\
\hline Characteristic & NCDS & NSHD & $\begin{array}{l}\text { Whitehall } \\
\text { II }\end{array}$ & CaPS & ELSA & HCS & Boyd Orr & LBC1921 & Total \\
\hline Number of participants & 7,363 & 2,674 & 3,143 & 1,318 & 5,612 & 2,893 & 397 & 516 & 23,916 \\
\hline Male, $\%$ & 50 & 50 & 77 & 100 & 46 & 53 & 45 & 41 & 55 \\
\hline $\begin{array}{l}\text { Age }^{\mathrm{a}} \text { in years, median } \\
\text { (range) }\end{array}$ & 44 & 53 & $59(50-73)$ & $\begin{array}{c}61(52- \\
71)\end{array}$ & $\begin{array}{c}65(52- \\
90+)\end{array}$ & $\begin{array}{c}66(59- \\
73)\end{array}$ & $\begin{array}{c}70(64- \\
82)\end{array}$ & $\begin{array}{c}79(77- \\
80)\end{array}$ & $\begin{array}{r}56(44- \\
90+)\end{array}$ \\
\hline$A P O E \varepsilon 4$ carrier status ${ }^{\mathrm{b}}, \%$ & 27 & 28 & 26 & 26 & 26 & 26 & 27 & 24 & 27 \\
\hline \multicolumn{10}{|l|}{ LDL-C-raising allele frequency } \\
\hline rs515135 $A P O B, \mathrm{C}$ & 0.82 & 0.82 & 0.82 & 0.82 & 0.82 & 0.82 & 0.82 & 0.85 & 0.82 \\
\hline rs $2228671 L D L R, \mathrm{C}$ & 0.87 & 0.88 & 0.87 & 0.88 & 0.88 & 0.88 & 0.88 & 0.88 & 0.87 \\
\hline rs629301 SORT1, A & 0.78 & 0.77 & 0.79 & 0.77 & 0.78 & 0.78 & 0.77 & 0.75 & 0.78 \\
\hline
\end{tabular}

Number of participants represents those with available data for at least one phenotype and at least one genotype

CaPS Caerphilly Prospective Study, ELSA English Longitudinal Study of Ageing, HCS Hertfordshire Cohort Study, LBC1921 Lothian Birth Cohort 1921, NCDS National Child Development Study, NSHD National Survey of Health and Development

${ }^{a}$ Age at phase from which the majority of variables are taken, i.e. Boyd Orr: III; CaPS: III; ELSA: II; HAS: I; HCS: I; LBC1921: I; NCDS: Biomedical Survey (2002); NSHD: 1999 Collection; Whitehall II: VII

${ }^{\mathrm{b}} \varepsilon 4$ carrier defined as $\varepsilon 3 / \varepsilon 4$ or $\varepsilon 4 / \varepsilon 4 ; \varepsilon 2 / \varepsilon 4$ excluded from denominator

heterogeneity $=0.009$; Figure S5). There was no evidence for heterogeneity between men and women for other outcomes ( $p$ values $>0.1$; data not shown). For the few studies with data available, there was no strong evidence that the $\varepsilon 4$ allele was associated with cognitive decline within the time periods investigated (Table S3).

Associations between $A P O B, L D L R$ and SORT1 genotypes and cognitive capability

There was some evidence that the LDL-C-raising allele of rs2228671 ( $L D L R)$ was associated with lower semantic fluency scores (Table 2, Figure S6). There was no evidence for any other associations between the SNPs and measures of cognitive capability (Table 2). Table S4 that shows there was no evidence for associations between any of the measures and the allelic count of the number of LDL-C-raising and $\varepsilon 4$ alleles. There was no evidence for differences in genotypic effects by sex for the SNPs ( $p$ values $>0.06$; data not shown).

Associations between $A P O E$ genotypes and physical capability

Figures S7 to S10 show that there was no strong evidence of associations in the pooled analyses between the physical capability measures and $\varepsilon 2+$ and $\varepsilon 4+$ adjusting for age and sex, compared with $\varepsilon 3 / \varepsilon 3$ in the cross-sectional analyses. Within studies, there was evidence in ELSA that participants with $\varepsilon 4+$ had slower chair rise times than those with $\varepsilon 3 / \varepsilon 3$ (Figure S9). There was no evidence that carriers of $\varepsilon 4$ had poorer performance than noncarriers in the pooled analyses (Table 3 ). Table S5 shows that there was no evidence for interactions between the $\varepsilon 4$ allele and age within the studies. There was no evidence for heterogeneity between men and women in the effects of the $\varepsilon 4$ allele for these measures ( $p$ values $>0.6$; data not shown). In the longitudinal analyses, there was evidence in ELSA that the $\varepsilon 4$ allele was associated with decline in timed walk speed $(p$ value $=0.02$; Table S6).

Associations between $A P O B, L D L R$ and SORT1 genotypes and physical capability

Table 3 shows that there was no evidence for associations between the SNPs and measures of physical capability. Table S7 shows that there was evidence for associations between the allelic count of the number of LDL$\mathrm{C}$-raising and $\varepsilon 4$ alleles and poorer timed chair rises ( $p$ value $=0.01$ ). There was some evidence that the effect of the $\mathrm{C}$ allele of rs515135 (APOB) on timed chair rises differed by sex, with an inverse association observed in females only ( $p$ value for heterogeneity $=0.01$; Figure S11). There was also evidence for heterogeneity by sex for the effects of rs2228671 ( $L D L R)$ on ability to balance ( $p$ value for heterogeneity $=0.04$; Figure S12). 
Table 2 Summary of pooled associations between LDL-C-related genotypes and cognitive capability

\begin{tabular}{|c|c|c|c|c|c|}
\hline Measure & Genotype & Beta $(95 \% \mathrm{CI})$ & $p$ & $I^{2} \%$; Het $p$ & $N$ \\
\hline & $A P O E \varepsilon 4$ & $-0.01(-0.06-0.04)$ & 0.67 & $46.0 ; 0.14$ & 17,513 \\
\hline & rs515135 $A P O B$ & $-0.02(-0.04-0.01)$ & 0.23 & $0.0 ; 0.67$ & 16,473 \\
\hline \multirow[t]{3}{*}{ Word recall } & rs2228671 LDLR & $0.01(-0.02-0.04)$ & 0.49 & $0.0 ; 0.97$ & 16,481 \\
\hline & rs629301 SORT1 & $0.01(-0.02-0.03)$ & 0.52 & $0.0 ; 0.97$ & 16,494 \\
\hline & $A P O E \varepsilon 4$ & $-0.01(-0.16-0.14)$ & 0.89 & $53.6 ; 0.14$ & 3,528 \\
\hline \multirow[t]{4}{*}{ Phonemic fluency } & rs515135 $A P O B$ & $0.00(-0.07-0.07)$ & 0.96 & $7.8 ; 0.30$ & 3,638 \\
\hline & rs2228671 LDLR & $-0.03(-0.10-0.05)$ & 0.52 & $6.8 ; 0.30$ & 3,638 \\
\hline & rs629301 SORT1 & $-0.01(-0.06-0.05)$ & 0.83 & $0.0 ; 0.88$ & 3,636 \\
\hline & $A P O E \varepsilon 4$ & $-0.02(-0.05-0.02)$ & 0.31 & $0.0 ; 0.98$ & 18,796 \\
\hline \multirow[t]{4}{*}{ Semantic fluency } & rs515135 $A P O B$ & $-0.02(-0.05-0.01)$ & 0.16 & $0.0 ; 0.97$ & 17,761 \\
\hline & rs2228671 LDLR & $-0.03(-0.06-0.00)$ & 0.048 & $0.0 ; 0.51$ & 17,766 \\
\hline & rs629301 SORT1 & $0.01(-0.04-0.05)$ & 0.83 & $66.8 ; 0.02$ & 17,774 \\
\hline & $A P O E \varepsilon 4$ & $0.00(-0.05-0.06)$ & 0.91 & $54.6 ; 0.11$ & 14,348 \\
\hline \multirow[t]{3}{*}{ Search speed } & rs515135 $A P O B$ & $-0.02(-0.05-0.01)$ & 0.26 & $0.0 ; 0.82$ & 13,229 \\
\hline & rs2228671 LDLR & $0.01(-0.02-0.05)$ & 0.54 & $0.0 ; 0.81$ & 13,235 \\
\hline & rs629301 SORT1 & $0.00(-0.05-0.05)$ & 0.90 & $64.7 ; 0.06$ & 13,250 \\
\hline
\end{tabular}

Coefficients based on $z$ scores and adjusted for age and sex. Coefficients for $A P O E \varepsilon 4+$ : carrier vs. non- $\varepsilon 4$ carrier; else per LDL-C-raising allele

Table 3 Summary of pooled associations between LDL-C-related genotypes and physical capability

\begin{tabular}{|c|c|c|c|c|c|}
\hline Measure & Genotype & Beta $(95 \% \mathrm{CI})$ & $p$ & $I^{2} \%$; Het $p$ & $N$ \\
\hline & $A P O E \varepsilon 4$ & $0.01(-0.02-0.05)$ & 0.42 & $30.7 ; 0.23$ & 10,646 \\
\hline \multirow[t]{3}{*}{ Grip strength } & rs515135 $A P O B$ & $0.00(-0.04-0.03)$ & 0.81 & $41.5 ; 0.16$ & 11,247 \\
\hline & rs2228671 LDLR & $0.01(-0.01-0.04)$ & 0.40 & $0.0 ; 0.65$ & 11,234 \\
\hline & rs629301 SORT1 & $-0.01(-0.03-0.02)$ & 0.61 & $34.1 ; 0.21$ & 11,242 \\
\hline \multirow[t]{4}{*}{ Get up and go/walk speed } & $A P O E \varepsilon 4$ & $-0.02(-0.07-0.04)$ & 0.57 & $7.8 ; 0.36$ & 6,810 \\
\hline & rs515135 $A P O B$ & $0.00(-0.05-0.06)$ & 0.90 & $30.6 ; 0.22$ & 7,291 \\
\hline & rs2228671 LDLR & $0.04(-0.06-0.13)$ & 0.47 & $67.6 ; 0.01$ & 7,277 \\
\hline & rs629301 SORT1 & $0.01(-0.03-0.04)$ & 0.73 & $0.0 ; 0.42$ & 7,281 \\
\hline \multirow[t]{5}{*}{ Timed chair rises } & $A P O E \varepsilon 4$ & $-0.04(-0.10-0.02)$ & 0.18 & $37.8 ; 0.20$ & 8,159 \\
\hline & rs515135 $A P O B$ & $-0.03(-0.07-0.01)$ & 0.11 & $0.0 ; 0.85$ & 8,601 \\
\hline & rs2228671 LDLR & $0.00(-0.05-0.04)$ & 0.86 & $2.4 ; 0.36$ & 8,598 \\
\hline & rs629301 SORT1 & $-0.02(-0.06-0.01)$ & 0.16 & $0.0 ; 0.47$ & 8,601 \\
\hline & Genotype & OR $(95 \% \mathrm{CI})$ & $p$ & $I^{2} \%$; Het $p$ & $n / N$ \\
\hline \multirow[t]{4}{*}{ Ability to balance $\geq 5 \mathrm{~s}$} & $A P O E \varepsilon 4$ & $1.02(0.88-1.17)$ & 0.80 & $0.0 ; 0.63$ & $8,696 / 10,114$ \\
\hline & rs515135 $A P O B$ & $1.01(0.91-1.13)$ & 0.86 & $0.0 ; 0.67$ & $9,131 / 10,654$ \\
\hline & rs2228671 LDLR & $1.04(0.92-1.18)$ & 0.54 & $0.0 ; 0.70$ & $9,122 / 10,643$ \\
\hline & rs629301 SORT1 & $0.97(0.87-1.07)$ & 0.49 & $0.0 ; 0.92$ & $9,139 / 10,652$ \\
\hline
\end{tabular}

Coefficients for continuous outcomes based on $z$ scores. Adjusted for age and sex. Coefficients for $A P O E \varepsilon 4+$ : carrier vs. non- $\varepsilon 4$ carrier; else per LDL-C-raising allele 


\section{Discussion}

In this investigation of 23,916 adults aged between 44 and $90+$ from eight UK cohorts, we examined the genotypic effects of the $A P O E \varepsilon 2 / 3 / 4$ genotype on measures of cognitive and physical capability, as well as those of three other LDL-C-related genotypes: rs515135 $(A P O B), \mathrm{rs} 2228671(L D L R)$ and rs629301 (SORT1). For the four cognitive capability measures studied (word recall, phonemic fluency, semantic fluency and search speed), we found little evidence for associations with APOE genotype (Table 2). However, within-study investigations into interactions between $\varepsilon 4$ and age provided suggestive evidence for an adverse effect of $\varepsilon 4$ on word recall that increased with age ( $p$ value $=0.044, n=$ $4,971)$ as well as suggestive evidence for this on search speed in the largest age-heterogeneous cohort ( $p$ value $=$ $0.08, n=4,901$; Table S2). In the longitudinal analyses, we observed no evidence that the $\varepsilon 4$ allele was associated with cognitive decline. Investigations into the other genotypes revealed only borderline evidence for an association between the LDL-C-raising allele of rs2228671 $(L D L R)$ and reduced semantic fluency $(p$ value $=0.048, n=17,766$ ). There was little evidence to support associations between the APOE genotype and our physical capability measures (grip strength, timed get up and go/walk speed, timed chair rises and ability to balance) in the cross-sectional analysis and no evidence that genotypic effects vary by age. In the longitudinal analysis, there was evidence from ELSA that the $\varepsilon 4$ allele was associated with decline in timed walk speeds ( $p$ value $=0.02, n=2,195$; Table S6). We found little evidence for associations between the other LDL-Crelated genotypes and physical capability (Table 3).

Previous investigations into associations between the $A P O E$ genotype and measures of cognitive performance have produced mixed findings, possibly reflecting differences in cognitive domains and age (Wisdom et al. 2011). Meta-analyses of previous studies have found evidence for small adverse effects of the $\varepsilon 4$ allele on some cognitive domains, including episodic memory, as well as evidence that this effect may increase with age (Wisdom et al. 2011). An increasing effect with age has also been seen in a study of over 10,000 Korean older adults (Shin et al. 2014). However, no evidence was found for effects on measures on verbal ability (Wisdom et al. 2011). Results from our investigations on $A P O E$ are consistent with many of those findings, with evidence for an interaction between $\varepsilon 4$ and age on a measure of episodic memory and word recall and no evidence for any effects on our measures of verbal ability: semantic and phonemic fluency. Previous studies have observed a greater cognitive decline among carriers of the $\varepsilon 4$ allele compared with noncarriers; in particular, there is evidence from a middle-aged cohort for differences in delayed word recall over 6 years (Blair et al. 2005), and a longitudinal study showed that declines in memory scores may be apparent before 60 years (Davies et al. 2014). Our own investigation from the model in ELSA suggested a detectable difference from around 55 years. Fewer investigations have been reported on the associations between $A P O E$ and measures of physical performance; however, findings include no association with athletic performance (Tsianos et al. 2010) and associations between $\varepsilon 4$ and slower gait speeds from a study of 1,262 adults aged over 65 years (Melzer et al. 2005). Our larger investigation found evidence to support a role of $A P O E$ in decline in walk speeds over a 4-year period in one study but little evidence for associations with physical capability from cross-sectional analysis. Differences have been observed in the genotypic frequencies of rs11668477 in $L D L R$ for mild cognitive impairment status in a small study of 114 cases and 92 controls (Liu et al. 2012). We believe that this is the largest investigation between $A P O B, L D L R$ and SORT1 genotypes and measures of cognitive and physical performance.

Our large investigation into associations between the $A P O E$ genotype on cognitive capability measures provided only modest evidence for an adverse effect of the $\varepsilon 4$ allele. There was also little evidence from the three other investigated LDL-C-related genotypes, suggesting that the small effects of $A P O E$ observed previously on some cognitive measures in populationbased studies may not extend to genotypes implicated in LDL-C levels as a whole. However, the lack of evidence from our studies for these other SNPs may reflect a lack of power to observe possible small effects. Sample size calculations demonstrated around 11,000 individuals would be required to detect a beta coefficient of $0.05 \mathrm{z}$ score units with $80 \%$ power at the $5 \%$ significance level for a SNP such as rs515135 $(A P O B)$ using an observed minor allele frequency of 0.18 . We were sufficiently powered to detect differences as small as this for three of our cognitive measures; however, the observed pooled coefficients were much smaller. There are hypothesised mechanisms through which $A P O E$ may 
affect cognitive performance in non-demented adults other than through LDL-C, for instance, $\varepsilon 4 / \varepsilon 4$ has been associated with increased rate of hippocampal volume loss (Crivello et al. 2010) and increased white matter hyperintensity burden (Schilling et al. 2013). Furthermore, $A P O E$ has been associated with several other ageing phenotypes (Khan et al. 2013). Our results suggest that these mechanisms might not lead to cognitive differences at a population level. Similar to the reports on cognitive performance (Yaffe et al. 2002; Packard et al. 2007), associations between measures of LDL-C and physical performance in the literature have been mixed, with higher levels associated with slower walking speeds (Walston et al. 2002), but some evidence that it may be protective against frailty (Blain et al. 2012). Our findings from other LDL-C-related genotypes suggest that LDL-C may not be an important mechanism through which $A P O E$ could affect physical performance. However, other potential pathways include the modest effects on specific cognitive measures and their decline and effects on bone-related phenotypes, though evidence for the latter is inconsistent (Peter et al. 2011; Tolonen et al. 2011).

Despite the size of our study, large age range and the inclusion of other LDL-C-related genotypes, there are some limitations. First, our ability to detect effects of our genotypes on decline in cognitive and physical capability may have been hindered by generally short intervals between measures in the cohorts (often around four years). Second, despite the important cognitive measures included here, there may be other cognitive domains which may be influenced by the $A P O E$ genotype. Third, although we only observed modest evidence for a detrimental impact of the $\varepsilon 4$ allele on cognitive outcomes, it is possible that an effect may be influenced a likely higher proportion of preclinical dementia cases among the $\varepsilon 4$ carriers. However, it is possible that $A P O E$ also affects non-pathological cognitive ageing (Davies et al. 2014). Fourth, we did not conduct investigations into the differential effects among each of the six $A P O E$ genotypes; however, given we observed only modest evidence for differences between $\varepsilon 4$ and non- $\varepsilon 4$ carriers, it is unlikely that this study would have been sufficiently powered to observe any of the putatively smaller differences. Furthermore, larger meta-analyses were unable to detect differences between the effects of $\varepsilon 3 / \varepsilon 4$ and $\varepsilon 4 / \varepsilon 4$ on several cognitive domains (Wisdom et al. 2011).

\section{Conclusion}

This large, multi-cohort investigation into associations between $A P O E$ \&2/3/4 genotype, three other LDL-Crelated SNPs and measures of cognitive and physical capability provided little support for anything but modest effects of these genotypes on these traits in middleaged to older adults.

Acknowledgments We thank Kate Birnie, Vanessa Cox, Philip Curran, Nikki Graham, Karen Jameson, Jon Johnson, Aida Sanchez, Imran Shah, Andrew Taylor and Andrew Wong for providing the data. We acknowledge the support of Medical Research Council and Arthritis Research (UK). The Boyd Orr DNA bank was funded by the Wellcome Trust (Grant number: GR068468MA). Follow-up of the Boyd Orr cohort was supported by grants from the Wellcome Trust, World Cancer Research Fund, Research into Ageing and the British Heart Foundation. The Caerphilly Prospective study was conducted by the former MRC Epidemiology Unit (South Wales) and funded by the Medical Research Council of the United Kingdom. The Department of Social and Community Medicine, University of Bristol now maintains the archive. Samples were from the English Longitudinal Study of Ageing (ELSA) DNA Repository (EDNAR) and received support under a grant (AG1764406S1) awarded by the National Institute on Ageing (NIA). ELSA was developed by a team of researchers based at the National Centre for Social Research, University College London and the Institute of Fiscal Studies. The data were collected by the National Centre for Social Research. The Hertfordshire Cohort Study was conducted by the MRC Lifecourse Epidemiology Unit at the University of Southampton and funded by the Medical Research Council and the University of Southampton. We thank the Lothian Birth Cohort 1921 participants. We thank the Scottish Council for Research in Education for allowing access to the Scottish Mental Survey 1932. The Biotechnology and Biological Sciences Research Council (BBSRC) funded the phenotypic data collection and DNA preparation (project grant 15/SAG09977) and GWAS (project grant BB/ F019394/1). The work was undertaken by The University of Edinburgh Centre for Cognitive Ageing and Cognitive Epidemiology, part of the cross council Lifelong Health and Wellbeing Initiative (Centre grant G0700704/84698). Funding from the BBSRC, Engineering and Physical Sciences Research Council (EPSRC), Economic and Social Research Council (ESRC) and Medical Research Council (MRC) is gratefully acknowledged. The MRC NSHD is funded by the UK Medical Research Council. NCDS 1958 British birth cohort: phenotype measures (blood pressure, etc.) and DNA collection were funded by the MRC (project grant G0000934) and creation of immortalised cell lines by Wellcome Trust Grant $068545 / \mathrm{Z} / 02$. The NCDS cohort is managed by the Centre for Longitudinal Studies, Department of Quantitative Social Sciences, Institute of Education, London, which is funded as an ESRC Resource Centre. The MRC Centre of Epidemiology for Child Health is funded by the MRC. Great Ormond Street Hospital /University College London, Institute of Child Health receives a proportion of funding from the Department of Health's National Institute of Health Research ('Biomedical Research Centres' funding). The Whitehall II funding were from the Medical Research Council (K013351); British Heart 
Foundation; Health and Safety Executive; Department of Health; National Heart Lung and Blood Institute (NHLBI, R01HL36310), US, NIH: National Institute on Aging (NIA, R01AG013196 and R01AG034454), US, NIH; Agency for Health Care Policy Research (HS06516) and the John D and Catherine T MacArthur Foundation Research Networks on Successful Midlife Development and Socio-economic Status and Health. DG is an NIHR Senior Investigator. RC received support from the HALCyon programme funded by the New Dynamics of Ageing (RES-35325-0001). RC, DK and RH are supported by the UK Medical Research Council. Mi.K. is supported by the UK MRC, NHLBI, NIA, NIH US and a professorial fellowship from the Economic and Social Research Council. Me.K is supported by NHLBI, NIH (HL36310). TA is an ESRC PhD student. The HALCyon study team also includes Catharine Gale, James Goodwin, Alison Lennox, Marcus Richards, Thomas von Zglinicki, John Gallacher, Gita Mishra, Paul Shiels, Humphrey Southall, Andrew Steptoe, Panos Demakakos, Kate Tilling, Lawrence Whalley, Geraldine McNeill, Leone Craig, Carmen Martin-Ruiz, Paula Aucott, Emily Murray, Zeinab Mulla, Mike Gardner and Sam Parsons.

Conflict of interest The authors declare no competing interests.

Open Access This article is distributed under the terms of the Creative Commons Attribution License which permits any use, distribution, and reproduction in any medium, provided the original author(s) and the source are credited.

\section{References}

Anthopoulos PG, Hamodrakas SJ, Bagos PG (2010) Apolipoprotein $\mathrm{E}$ polymorphisms and type 2 diabetes: a meta-analysis of 30 studies including 5423 cases and 8197 controls. Mol Genet Metab 100:283-291. doi:10.1016/j. ymgme.2010.03.008

Aulchenko YS, Ripatti S, Lindqvist I et al (2009) Loci influencing lipid levels and coronary heart disease risk in 16 European population cohorts. Nat Genet 41:47-55. doi:10.1038/ng.269

Bennet AM, Di Angelantonio E, Ye Z et al (2007) Association of apolipoprotein E genotypes with lipid levels and coronary risk. JAMA 298:1300-1311. doi:10.1001/jama.298.11.1300

Bertram L, McQueen MB, Mullin K et al (2007) Systematic metaanalyses of Alzheimer disease genetic association studies: the AlzGene database. Nat Genet 39:17-23. doi:10.1038/ng1934

Blain H, Jaussent A, Béziat S et al (2012) Low serum IL-6 is associated with high 6-minute walking performance in asymptomatic women aged 20 to 70 years. Exp Gerontol 47:143-148. doi:10.1016/j.exger.2011.11.008

Blair CK, Folsom AR, Knopman DS et al (2005) APOE genotype and cognitive decline in a middle-aged cohort. Neurology 64: 268-276. doi:10.1212/01.WNL.0000149643.91367.8A

Bopp KL, Verhaeghen P (2005) Aging and verbal memory span: a meta-analysis. J Gerontol B Psychol Sci Soc Sci 60:P223233
Borkowski JG, Benton AL, Spreen O (1967) Word fluency and brain damage. Neuropsychologia 5:135-140. doi:10.1016/ 0028-3932(67)90015-2

Brown M, Dodgeon B (2010) NCDS cognitive assessments at age 50: initial results. CLS Working Paper 2010/1

Bunce D, Anstey KJ, Burns R et al (2011) Does possession of apolipoprotein $\mathrm{E} \varepsilon 4$ benefit cognitive function in healthy young adults? Neuropsychologia 49:1693-1697. doi:10. 1016/j.neuropsychologia.2011.02.042

Caselli RJ, Dueck AC, Osborne D et al (2009) Longitudinal modeling of age-related memory decline and the APOE epsilon4 effect. N Engl J Med 361:255-263. doi:10.1056/ NEJMoa0809437

Clouston SAP, Brewster P, Kuh D et al (2013) The dynamic relationship between physical function and cognition in longitudinal aging cohorts. Epidemiol Rev. doi:10.1093/epirev/mxs004

Cooper R, Hardy R, Aihie Sayer A et al (2011) Age and gender differences in physical capability levels from mid-life onwards: the harmonisation and meta-analysis of data from eight UK cohort studies. PLoS ONE 6:e27899. doi:10. 1371/journal.pone.0027899

Cordell HJ, Clayton DG (2005) Genetic association studies. Lancet 366:1121-1131. doi:10.1016/S0140-6736(05)67424-7

Crivello F, Lemaitre H, Dufouil C et al (2010) Effects of ApoEepsilon4 allele load and age on the rates of grey matter and hippocampal volumes loss in a longitudinal cohort of 1186 healthy elderly persons. Neuroimage 53:1064-1069. doi:10. 1016/j.neuroimage.2009.12.116

Csuka M, McCarty DJ (1985) Simple method for measurement of lower extremity muscle strength. Am J Med 78:77-81

Davies G, Harris SE, Reynolds CA et al (2014) A genome-wide association study implicates the APOE locus in nonpathological cognitive ageing. Mol Psychiatry 19:7687. doi:10.1038/mp.2012.159

Deary IJ, Whiteman MC, Pattie A et al (2004a) Apolipoprotein e gene variability and cognitive functions at age 79: a followup of the Scottish mental survey of 1932. Psychol Aging 19: 367-371. doi:10.1037/0882-7974.19.2.367

Deary IJ, Whiteman MC, Starr JM et al (2004b) The impact of childhood intelligence on later life: following up the Scottish mental surveys of 1932 and 1947. J Pers Soc Psychol 86: 130-147. doi:10.1037/0022-3514.86.1.130

Deary IJ, Whalley LJ, Batty GD, Starr JM (2006) Physical fitness and lifetime cognitive change. Neurology 67:1195-1200. doi:10.1212/01.wnl.0000238520.06958.6a

Deary IJ, Gow AJ, Pattie A, Starr JM (2012) Cohort profile: the Lothian Birth Cohorts of 1921 and 1936. Int J Epidemiol 41: 1576-1584. doi:10.1093/ije/dyr197

Gauderman W, Morrison J (2006) QUANTO 1.1: A computer program for power and sample size calculations for geneticepidemiology studies, [http://hydra.usc.edu/gxe/]

Higgins JPT, Thompson SG, Deeks JJ, Altman DG (2003) Measuring inconsistency in meta-analyses. BMJ 327:557560. doi:10.1136/bmj.327.7414.557

Houlihan LM, Davies G, Tenesa A et al (2010) Common variants of large effect in F12, KNG1, and HRG are associated with activated partial thromboplastin time. Am J Hum Genet 86: 626-631. doi:10.1016/j.ajhg.2010.02.016

Hyman BT, Gomez-Isla T, Briggs M et al (1996) Apolipoprotein E and cognitive change in an elderly population. Ann Neurol 40:55-66. doi:10.1002/ana.410400111 
Ihle A, Bunce D, Kliegel M (2012) APOE $\varepsilon 4$ and cognitive function in early life: a meta-analysis. Neuropsychology 26 : 267-277. doi:10.1037/a0026769

Johnson W, Deary IJ, McGue M, Christensen K (2009) Genetic and environmental links between cognitive and physical functions in old age. J Gerontol B Psychol Sci Soc Sci 64: 65-72. doi:10.1093/geronb/gbn033

Kelley GA, Kelley KS, Roberts S, Haskell W (2012) Comparison of aerobic exercise, diet or both on lipids and lipoproteins in adults: a meta-analysis of randomized controlled trials. Clin Nutr 31:156-167. doi:10.1016/j.clnu.2011.11.011

Khan TA, Shah T, Prieto D et al (2013) Apolipoprotein E genotype, cardiovascular biomarkers and risk of stroke: systematic review and meta-analysis of 14,015 stroke cases and pooled analysis of primary biomarker data from up to 60 , 883 individuals. Int J Epidemiol 42:475-492. doi:10.1093/ ije/dyt034

Kuh D, Bassey EJ, Butterworth S et al (2005) Grip strength, postural control, and functional leg power in a representative cohort of British men and women: associations with physical activity, health status, and socioeconomic conditions. J Gerontol A Biol Sci Med Sci 60:224-231

Kulminski A, Ukraintseva SV, Arbeev KG et al (2008) Association between APOE epsilon 2/epsilon 3/epsilon 4 polymorphism and disability severity in a national longterm care survey sample. Age Ageing 37:288-293. doi:10. 1093/ageing/afn003

Law MR, Wald NJ, Rudnicka AR (2003) Quantifying effect of statins on low density lipoprotein cholesterol, ischaemic heart disease, and stroke: systematic review and meta-analysis. BMJ 326:1423. doi:10.1136/bmj.326.7404.1423

Lill C, Roehr J The PDGene Database. Alzheimer Research Forum. Available at: http://www.pdgene.org/

Little J, Higgins JPT, Ioannidis JPA et al (2009) Strengthening the reporting of genetic association studies (STREGA): an extension of the STROBE statement. Eur J Epidemiol 24:3755. doi:10.1007/s10654-008-9302-y

Liu X, Yue C, Xu Z et al (2012) Association study of candidate gene polymorphisms with amnestic mild cognitive impairment in a Chinese population. PLoS ONE 7:e41198. doi:10. 1371/journal.pone.0041198

Mahley RW, Rall SC Jr (2000) Apolipoprotein E: far more than a lipid transport protein. Annu Rev Genomics Hum Genet 1: 507-537. doi:10.1146/annurev.genom.1.1.507

Marmot M, Brunner E (2005) Cohort Profile: the Whitehall II study. Int J Epidemiol 34:251-256. doi:10.1093/ije/dyh372

Marmot M, Banks J, Blundell R, et al. (2003) Health, wealth and lifestyles of the older population in England: ELSA 2002

Martin RM, Gunnell D, Pemberton J et al (2005) Cohort profile: The Boyd Orr cohort - an historical cohort study based on the 65 year follow-up of the Carnegie Survey of Diet and Health (1937-39). Int J Epidemiol 34:742-749. doi:10.1093/ije/dyi124

Matteini AM, Fallin MD, Kammerer CM et al (2010) Heritability estimates of endophenotypes of long and health life: the Long Life Family Study. J Gerontol A Biol Sci Med Sci 65:13751379. doi:10.1093/gerona/glq154

McKay GJ, Silvestri G, Chakravarthy U et al (2011) Variations in apolipoprotein $\mathrm{E}$ frequency with age in a pooled analysis of a large group of older people. Am J Epidemiol 173:13571364. doi:10.1093/aje/kwr015
Melzer D, Dik MG, van Kamp GJ et al (2005) The apolipoprotein $\mathrm{E}$ e4 polymorphism is strongly associated with poor mobility performance test results but not selfreported limitation in older people. J Gerontol A Biol Sci Med Sci 60:1319-1323

Nebel A, Kleindorp R, Caliebe A et al (2011) A genome-wide association study confirms APOE as the major gene influencing survival in long-lived individuals. Mech Ageing Dev 132:324-330. doi:10.1016/j.mad.2011.06.008

Packard CJ, Westendorp RGJ, Stott DJ et al (2007) Association between apolipoprotein E4 and cognitive decline in elderly adults. J Am Geriatr Soc 55:1777-1785. doi:10.1111/j.15325415.2007.01415.x

Peter I, Crosier MD, Yoshida M et al (2011) Associations of APOE gene polymorphisms with bone mineral density and fracture risk: a meta-analysis. Osteoporos Int 22:1199-1209. doi:10.1007/s00198-010-1311-5

Podsiadlo D, Richardson S (1991) The timed "Up \& Go": a test of basic functional mobility for frail elderly persons. J Am Geriatr Soc 39:142-148

Power C, Elliott J (2006) Cohort profile: 1958 British birth cohort (National Child Development Study). Int J Epidemiol 35:34 41. doi:10.1093/ije/dyi183

Richards M, Kuh D, Hardy R, Wadsworth M (1999) Lifetime cognitive function and timing of the natural menopause. Neurology 53:308-314

Riley RD, Lambert PC, Abo-Zaid G (2010) Meta-analysis of individual participant data: rationale, conduct, and reporting. BMJ 340:c221

Rockwood K, Nassar B, Mitnitski A (2008) Apolipoprotein Epolymorphism, frailty and mortality in older adults. J Cell Mol Med 12:2754-2761. doi:10.1111/j.1582-4934.2008. 00270.x

Sabia S, Kivimaki M, Kumari M et al (2010) Effect of apolipoprotein E epsilon4 on the association between health behaviors and cognitive function in late midlife. Mol Neurodegener 5:23. doi:10.1186/1750-1326-5-23

Schiepers OJG, Harris SE, Gow AJ et al (2012) APOE E4 status predicts age-related cognitive decline in the ninth decade: longitudinal follow-up of the Lothian Birth Cohort 1921. Mol Psychiatry 17:315-324. doi:10.1038/mp.2010.137

Schilling S, DeStefano AL, Sachdev PS et al (2013) APOE genotype and MRI markers of cerebrovascular disease: systematic review and meta-analysis. Neurology 81:292-300. doi:10. 1212/WNL.0b013e31829bfda4

Shin M-H, Kweon S-S, Choi J-S et al (2014) The effect of an APOE polymorphism on cognitive function depends on age. J Neurol 261:66-72. doi:10.1007/s00415-013-7157-y

Sport C of EC for the D of (1993) EUROFIT: Handbook for the EUROFIT Tests of Physical Fitness. Strasbourg

Stevens KN, Lang IA, Guralnik JM, Melzer D (2008) Epidemiology of balance and dizziness in a national population: findings from the English Longitudinal Study of Ageing. Age Ageing 37:300-305. doi:10.1093/ageing/afn019

Stroup DF, Berlin JA, Morton SC et al (2000) Meta-analysis of observational studies in epidemiology: a proposal for reporting. Meta-analysis Of Observational Studies in Epidemiology (MOOSE) group. JAMA 283:2008-2012

Syddall HE, Aihie Sayer A, Dennison EM et al (2005) Cohort profile: the Hertfordshire cohort study. Int J Epidemiol 34: 1234-1242. doi:10.1093/ije/dyi127 
Taylor AE, Guthrie PAI, Smith GD et al (2011) IQ, educational attainment, memory and plasma lipids: associations with apolipoprotein E genotype in 5995 children. Biol Psychiatry 70:152-158. doi:10.1016/j.biopsych.2010.10.033

Teslovich TM, Musunuru K, Smith AV et al (2010) Biological, clinical and population relevance of 95 loci for blood lipids. Nature 466:707-713. doi:10.1038/nature09270

Tolonen S, Mikkilä V, Laaksonen M et al (2011) Association of apolipoprotein E promoter polymorphisms with bone structural traits is modified by dietary saturated fat intake - the cardiovascular risk in Young Finns study. Bone 48:10581065. doi:10.1016/j.bone.2011.01.013

Tsianos GI, Evangelou E, Boot A et al (2010) Associations of polymorphisms of eight muscle- or metabolism-related genes with performance in Mount Olympus marathon runners. J Appl Physiol 108:567-574. doi:10.1152/japplphysiol.00780.2009

Vasunilashorn S, Glei DA, Lin Y-H, Goldman N (2013) Apolipoprotein $\mathrm{E}$ and measured physical and pulmonary function in older Taiwanese adults. Biodemography Soc Biol 59:57-67. doi:10.1080/19485565.2013.778703

Wadsworth M, Kuh D, Richards M, Hardy R (2006) Cohort Profile: The 1946 National Birth Cohort (MRC National Survey of Health and Development). Int J Epidemiol 35: 49-54. doi:10.1093/ije/dyi201
Walston J, McBurnie MA, Newman A et al (2002) Frailty and activation of the inflammation and coagulation systems with and without clinical comorbidities: results from the Cardiovascular Health Study. Arch Intern Med 162:23332341

Wellcome Trust Case Control Consortium (2007) Genome-wide association study of 14,000 cases of seven common diseases and 3,000 shared controls. Nature 447:661-678. doi:10. 1038/nature05911

Wilson PW, D'Agostino RB, Levy D et al (1998) Prediction of coronary heart disease using risk factor categories. Circulation 97:1837-1847

Wisdom NM, Callahan JL, Hawkins KA (2011) The effects of apolipoprotein $\mathrm{E}$ on non-impaired cognitive functioning: a meta-analysis. Neurobiol Aging 32:63-74. doi:10.1016/j. neurobiolaging.2009.02.003

Yaffe K, Barrett-Connor E, Lin F, Grady D (2002) Serum lipoprotein levels, statin use, and cognitive function in older women. Arch Neurol 59:378-384

Zhao JH, Brunner EJ, Kumari M et al (2005) APOE polymorphism, socioeconomic status and cognitive function in midlife-the Whitehall II longitudinal study. Soc Psychiatry Psychiatr Epidemiol 40:557-563. doi:10.1007/s00127-0050925-y 